\title{
DSTiPE Algorithm for Fuzzy Spatio-Temporal Risk Calculation in Wireless Environments
}

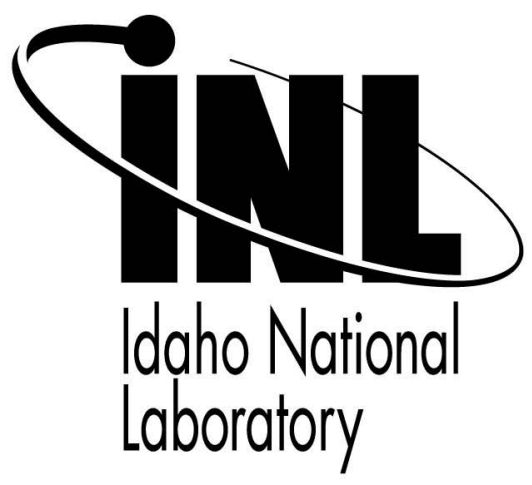

\author{
Kurt Derr \\ Milos Manic
}

September 2008 


\section{DSTiPE Algorithm for Fuzzy Spatio-Temporal Risk Calculation in Wireless Environments}

\author{
Kurt Derr, Todd Vollmer \\ Idaho National Laboratory \\ 2525 Freemont Avenue \\ Idaho Falls, ID 83415, USA \\ derr5843@uidaho.edu, Todd.Vollmer@inl.gov
}

\author{
Milos Manic \\ University of Idaho at Idaho Falls \\ 1776 Science Center Dr., Suite 306 \\ Idaho Falls, ID 83402, USA \\ misko@ieee.org
}

\begin{abstract}
Time and location data play a very significant role in a variety of factory automation scenarios, such as automated vehicles and robots, their navigation, tracking, and monitoring, to services of optimization and security. Pervasive wireless capabilities combined with time and location information are enabling new applications in areas such as transportation systems, health care, elder care, military, emergency response, critical infrastructure, and law enforcement.

A wireless object in proximity to some area for a duration of time may pose a risk hazard to the environment. This paper presents a novel fuzzy based spatio-temporal risk calculation DSTiPE method that a wireless object may present to the environment. The presented Matlab based application for cluster extraction is verified on a diagonal vehicle movement example.
\end{abstract}

\section{Introduction}

Numerous problems benefit from analysis of both spatial and temporal data. Wireless communications in factory automation is the medium for collecting spatial and temporal information. Distributed control systems, sensors, actuators, controllers, autonomous guided vehicles and truck fleets all may use wireless communications $[1,2]$. Factory automation applications using spatio-temporal data include distributed factory management, autonomous guided vehicles, event correlation, supply chain management, automating production processes, process optimization and scheduling, radio frequency identification (RFID) systems for tagging goods in a factory floor automation system, and automated barcode systems [3,4,5].

Autonomous vehicles $[6,7]$ require location based data for navigation. Cybernetic transportation systems are in use today at Schiphol airport in Amsterdam, Rotterdam Netherlands for travel between a train station and office complex, and at Heathrow airport in London later this year [8]. The integration of both spatial and temporal data enables vehicles to optimize their navigation to minimize fuel consumption and/or travel time. A central transportation management system can also use spatial and temporal information to ensure a good redistribution of vehicles at the fleet level matching supply and demand while minimizing risk.

Spatio-temporal patterns enable the detection of significant activities, places, and events as well as provide useful information for optimization in mobility applications. A significant place is an area, at some level of granularity, where an object/person spends an amount of time exceeding a predefined threshold $t$. The spatiotemporal characteristics of some device allow us to infer behavior as well as ascertain the degree of risk that some object/person may present to the environment, themselves, or others; e.g. automated vehicles delivering and picking up goods or staying for extended time periods at locations that pose a hazard to other objects/people, such as at street intersections, entrances to buildings, near critical infrastructure assets, or in specific areas within an automated factory;.

Other applications of spatio-temporal risk analysis include: intelligent transportation spaces $[9,10]$; smart hospitals [11]; optimization and security of equipment movement in military and commercial supply chains; real-time danger estimation to a fire fighter within a burning building; monitoring healthcare delivery in geriatric residents; tracking, monitoring, and navigation of vehicles, objects, robots, or people.

A time-based clustering algorithm is used to determine significant places of objects with wireless communications at multiple levels of granularity. Next, the time and distance measurements for the significant places are used in the fuzzy risk calculation as inputs. The output of the fuzzy risk calculation is a risk/danger factor of the person/object with wireless communications to the environment, other objects or people.

In this paper we present a novel fuzzy based risk calculation using a Dynamic Spatio-Temporal for Significant Place Extraction (DSTiPE) algorithm to determine the risk that an object with wireless capability presents to the environment. To the best of our knowledge this algorithmic approach has not been previously used for risk/danger estimation. The paper is organized as follows. Section 2 discusses related work, section 3 reviews the time-based clustering for significant place extraction algorithm, section 4 describes the fuzzy spatio-temporal risk calculations, and section 5 presents future work.

\section{Related Works}

Some companies such as Komatsu and Modular 
Mining Systems are providing driverless (autonomous) haul truck fleets to operate in mining operations [12]. Autonomous guided vehicles are important in factory automation as pallet trucks for transporting loads to and from the factory floor [13].

The Navitime project [14] supports urban pedestrian navigation services. Navitime computes itineraries from source to destination for several different types of transportation, walking, driving, trains, automobile, buses, taxis, and planes.

Spatio-temporal analysis of 911 call stream data is used to detect emergency events [15]. Calls received after an event and around the events location could be used to detect the emergency event, plan the local response, and facilitate resource response to potential disasters.

Place Lab data [16] has been used to extract places from traces of locations [17]. Specific distance and time threshold parameters are used with a time-based clustering approach to determine significant places.

The Real Time Rome project [18] uses spatial and temporal data to understand urban dynamics, such as how neighborhoods are used during the day, and how goods and services are distributed within a city. The goal of the project is to understand the present and anticipate the future of urban environments.

\section{Time-Based Clustering For Significant Place Extraction}

A place is a geographic area defined by a location(s), such as a public library, place of work, school, café, or other social gathering area. A location is defined as a set of coordinates. A place is significant if a person spends more than some period of time, $t$, at that area.

The Dynamic Spatio-Temporal for Significant Place Extraction (DSTiPE) algorithm uses a nearest-neighbor type distance based clustering algorithm to calculate the clusters [21]. Time is then used to determine whether the clusters are significant. Varying the clusters based on time, $t$, and distance, $d$, thresholds produces problem domain dependent results.

A set of significant places, $S$, is defined as:

$$
S=\left\{S_{1}, S_{2}, \ldots, S_{m}\right\}
$$

where $m$ is the number of significant places dynamically determined.

$$
S_{i}=f(d, t, L, T)
$$

A significant place is a function of $d, t$, a set of locations $L$, and a set of times $T$ for each location. A set of locations is defined as:

$$
L=\left\{L_{1}, L_{2}, \ldots, L_{n}\right\}
$$

where $n$ is the number of locations for which measurements have been taken. For each location there will be some measure of time, a stay, which specifies how much time a user/object has remained at that location $[19,20]$. The number of stays is equal to the number of location measurements.

$$
T=\left\{T_{1}, T_{2}, \ldots, T_{n}\right\}
$$

A cluster of locations represents locations within geographical proximity of one another based on the distance threshold $d$. No a priori knowledge of the number of clusters is needed in advance. Each cluster has a center of gravity, $C_{j}$, and a radius associated with it:

$$
\forall C_{j},\left(C O G_{j}, R_{j}\right), j=1,2, \ldots, p
$$

The Euclidean distance (dist) metric is used to determine membership in a cluster.

$$
\text { dist }=\sqrt{\left(x_{i}-x_{j}\right)^{2}+\left(y_{i}-y_{j}\right)^{2}}
$$

where $\left(x_{i}, y_{i}\right)$ represent a new location $L_{i}$ and $\left(x_{j}, y_{j}\right)$ represent the COG of the current cluster. $L_{i}$ becomes a member of the current cluster if dist $<d$, otherwise a new cluster is formed. The distance threshold, $d$, directly affects the number of locations in a cluster.

The time threshold, $t$, determines whether or not a cluster represents a significant place. The total time, $T_{C j}$, for a cluster $C_{j}$ is a summation of all $T_{i}$ that represents a stay for cluster $C_{j}$.

$$
T_{C_{j}}=\sum T_{i}, \forall T_{i} \in C_{j}
$$

The longer a person/object remains within a localized area the larger the time-based cluster. The DSTiPE timebased clustering algorithm assumes that location and time data have previously been collected. The DSTiPE algorithm then works as follows:

Step 1: The first cluster $C_{j}$ is initialized to the first location $L_{i}$.

Step 2: For each new location $L_{i}$, if $\operatorname{dist}\left(L_{i}, C O G_{j}\right)<\mathrm{d}$, then add $L_{i}$ to cluster $C_{j}$ and recompute $C O G_{j}$ $\left(C O G_{j}=\right.$ mean $\left(\right.$ all $L_{i}$ in $\left.C_{j}\right)$ ), else make $L_{i}$ the initial center of cluster $C_{j+1}$. Repeat step 2 for every location $L_{i}$.

Step 3: Determine significant locations based on time. For each cluster $C_{j}$, calculate the total time $T_{C_{j}}$ that patterns spend in a cluster $C_{j}$.

Step 3.1: If $T_{C_{j}}>t$, then this cluster of locations, $C_{j}$, represents a significant location. All tuples $\left(L_{i}, T_{j}\right)$ for cluster $C_{j}$ are added to significant place $S_{k}, k=k+1$.

Repeat step 3 for every cluster $\boldsymbol{C}_{\boldsymbol{j}}$.

Synthetic movement data patterns are used to demonstrate the algorithm due to the difficulty of obtaining real world data. Figure 1 represents a diagonal movement pattern across a grid area starting at $(1.5,3.5)$.

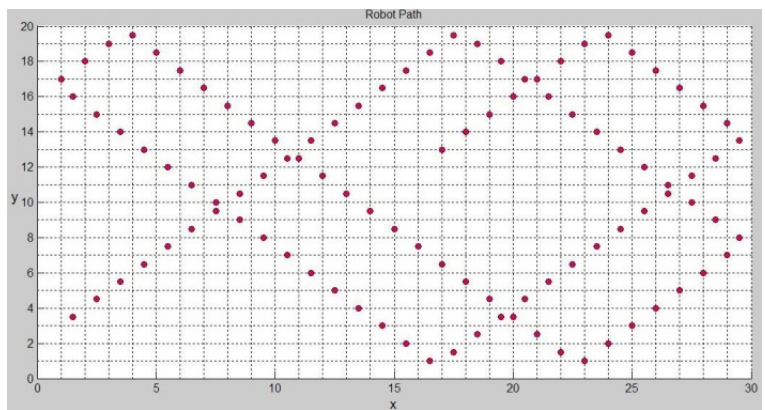

Figure 1. Diagonal Robot/Vehicle Movement

Figure 2 shows the MATLAB graphical user interface 
(GUI) for using the DSTiPE algorithm to determine

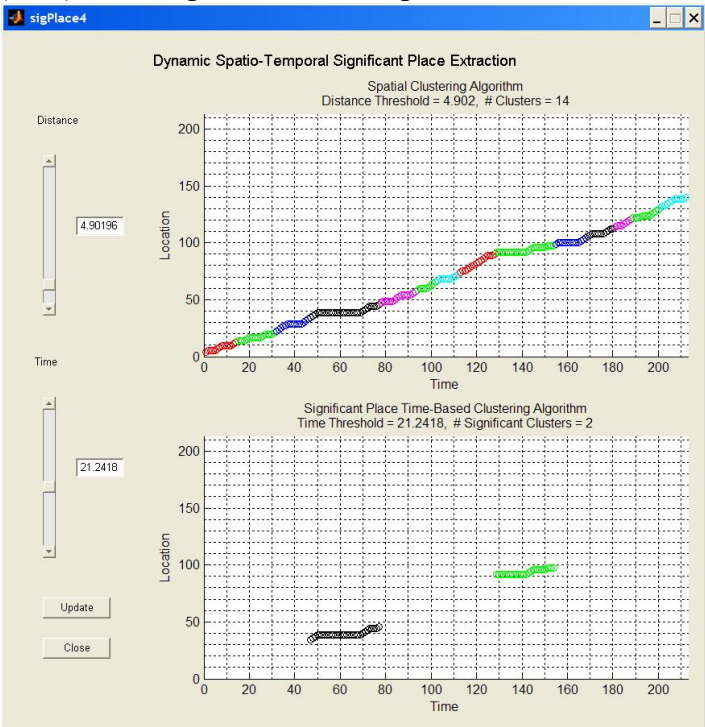

Figure 2. DSTiPE Algorithm with Diagonal Path

significant locations based on diagonal path and time. Distance and time thresholds are approximately 5 and 21, respectively. Time data for each location was randomly generated based on a stay probability. Only 2 of the 14 detected clusters satisfied the threshold criterion and as such qualified as significant places; i.e. $T_{C_{j}}>t$.

\section{Fuzzy Spatio-Temporal Risk Calculation}

The MATLAB fuzzy inference system editor was used to model input and output fuzzy sets and their rules. Both the fuzzy sets and fuzzy rules apply to each significant place $S_{i}$ data set determined by the DSTiPE algorithm. The model is based on using fuzzy representations of distance and time concepts (inputs) to calculate the degree of danger or risk (output) that some object/person poses to the environment or others. The fuzzy algorithm is evaluated using general modus ponens and max-min composition [22, 23, 24].

The shapes of both the input and output fuzzy sets will vary depending upon the problem domain. Fuzzy sets based on distance are shown in Figure 3. The fuzzy sets for the concept "distance" are close, middle, and far, which correspond to the circular areas in Figure 5 starting with the inner most circles.

The fuzzy set close represents a distance of 18 meters or less from the high value asset. Fuzzy sets based on "time" (low, medium, and high) are shown in Figure 4.

As an example of applying the concept of fuzzy spatiotemporal risk calculation, consider an office building with high-value assets with wireless location tracking tags located in the center of the building (blue area in the center of Figure 5).

This is an area of the building that is considered to be a significant place. The degree of danger that some object/person poses to these assets is based on the distance from the person to the assets and the amount of time that a person stays in different locations as they move about the building. The area within the smallest circle represents a close distance to the asset, between the first and second circles represents a medium distance, and outside the largest circle represents a far distance.

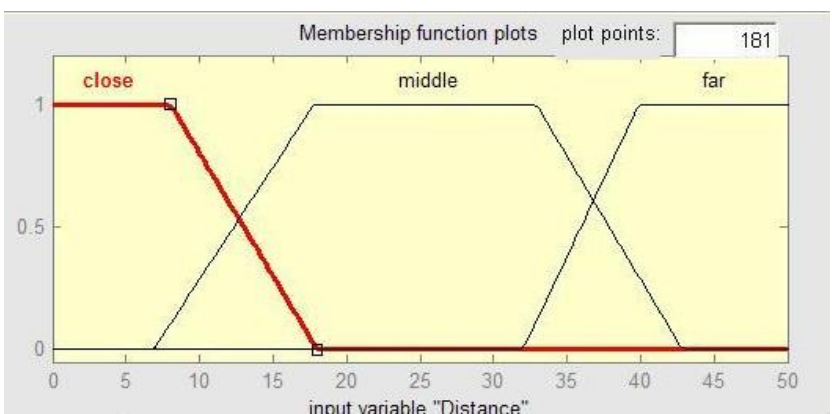

Figure 3. Input Fuzzy Sets for Distance

\section{Concept}

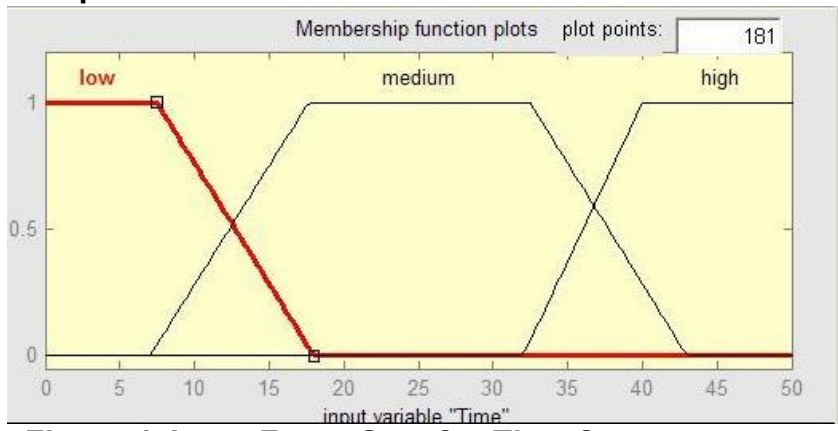

Figure 4. Input Fuzzy Sets for Time Concept

As the distance from some object/person to a significant place becomes closer and as the stay (time spent in some location) increases, so does the risk/danger. Figure 6 shows the output fuzzy sets (low, medium and high) for the concept of risk/danger. A degree of risk/danger of 0.855 is produced using the fuzzy rules produced from the distance and time fuzzy sets and the distance and time thresholds of 4.9 and 21.89 , respectively as shown in Figure 2 . The output is calculated using the membership functions and max-min composition as follows.

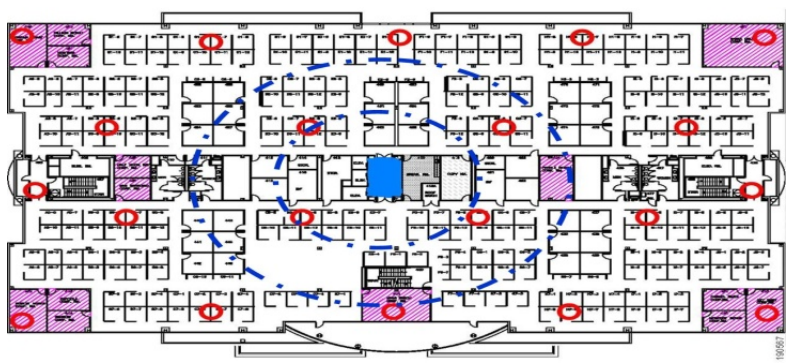

Figure 5. Building Offices Risk Areas

The degree of membership in trapezoidal membership functions is defined as:

$$
\mu_{A}=\max \left(\min \left(\frac{x-a}{b-a}, 1, \frac{d-x}{d-c}\right), 0\right)
$$

Where $\mathrm{x}=$ input, $a$ and $d$ are feet of trapezoid, and $b$ 
and $c$ are shoulders of the trapezoid.

The degree of membership in triangular membership functions is defined as:

$$
\mu_{B}=\max \left(\min \left(\frac{x-a}{b-a}, \frac{c-x}{c-b}\right), 0\right)
$$

Where $\mathrm{x}=$ input, $a$ and $c$ are feet of triangle, and $b$ is the peak.

The degree of fulfillment $(D O F)$ for each rule is a measure of similarity between the input and the antecedents of the rule.

$$
D O F_{i}=\min \left(\mu_{\text {dis } \tan c e}, \mu_{\text {time }}\right)
$$

The total fuzzy output is the union of all of the outputs for the rules that fire $(D O F>0)$.

$$
\mu_{\text {out }}=\max \left(D O F_{i}\right)
$$

Distance and time determine significant place. Risks of accidents, crime, terrorist activity, life/death situations, or other may vary dependent upon time, distance, and place.

\section{Future Work}

The fuzzy sets for risk calculation are problem domain dependent. Multiple types of risk calculation for the same problem domain may be valuable dependent upon context. Determining the fuzzy set for several real-world datasets will be one of future work directions.

An algorithmic approach for determining distance and time thresholds based on problem domain would add value to significant location estimation. Some mapping between a significant place label and significant location extraction would also make the results more meaningful.

While extracting significant places from location and time data has been done before, an adaptive approach based on the granularity of the problem (multi resolution) has been missing. This represents another area of future work.

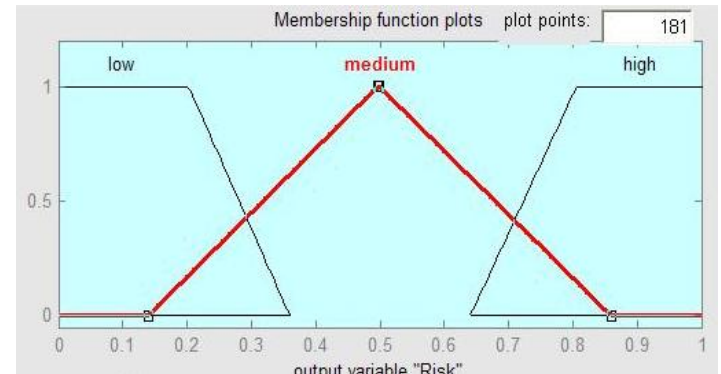

Figure 6. Output Fuzzy Sets for the Concept "Risk/Danger"

\section{References}

[1] U. Nunes, José A. Fonseca, L. Almeida, R. Araújo, R. Maia, "Fieldbuses in Real-Time Control of Autonomous Vehicles", ROBOTICA, Special Issue on Cost Effective Automation, Vol.22, Part 4, May 2003

[2] P. Neumann,"Communication in industrial automationWhat is going on?", ScienceDirect, CEP 15 (2007) pp.1332-1347

[3] A. Gupta, T. Brown, S. Seshasai, "24-Hour Knowledge Factory: Using Internet Technology to Leverage Spatial and Temporal Separations", ACM Transactions on Internet Technology, Vol. 7, Issue 3, August 2007

[4] E. Yoneki, J. Bacon, "A survey of Wireless Sensor Network technologies: research trends and middleware's role", University of Cambridge TR No. 646, Sept. 2005

[5] F. Pellegrini, D. Miorandi, S. Vitturi, A. Zanella, "On the Use of Wireless Networks at Low Level of Factory Automation Systems", IEEE Transactions On Industrial Informatics, Vol. 2, No. 2, May 2006

[6] C. Urmson, W. Whittaker, "Self-Driving Cars and the Urban Challenge", IEEE Intelligent Systems, March-April 2008, pp.66-68.

[7] M. Parent, "Advanced Urban Transport: Automation is on the Way", IEEE Intelligent Systems, March-April 2008, pp.9-11.

[8] D. Geske, "The Future for Autonomous Haul Trucks", Sept. 2004, http://findarticles.com/p/articles/mi_m0FZX/is_9_70/ai_n 7069121.

[9] L. Yang, F. Wang, "Driving into Intelligent Spaces with Pervasive Communications", IEEE Intelligent Systems, Jan.-Feb. 2007, pp.12-15.

[10] F. Wang, "Driving into the Future with ITS", IEEE Intelligent Systems, May-June 2006, pp.94-95.

[11] D. Sanchez, M. Tentori, J. Favela, "Activity Recognition for the Smart Hospital", IEEE Intelligent Systems, MarchApril 2008, pp.19-25.

[12] NonStop http://www.mmsi.com/autonomous.shtml

Haulage,

13] Autonomous Guided Vehicles, http://www.processregister.com/Autonomous_Guided_Ve hicle Pallet Truck/Suppliers/pid15754.htm

[14] M. Arikawa, S. Konomi, K. Ohnishi, "Navitime: Supporting Pedestrian Navigation in the Real World", IEEE Pervasive Computing, July-Sept. 2007, pp.21-29.

[15] W. Hodgkiss, et al, "Spatiotemporal Analysis of 9-1-1 Call Stream Data", Proceedings of the 2005 National Conference on Digital Government Research, May 15 18, 2005, pp: $293-294$.

[16] B. N. Schilit, et. al.,"Challenge: Ubiquitous LocationAware Computing and the Place Lab Initiative", WMASH September 2003.

[17] J.H. Kang, W. Welbourne, B. Stewart, G. Borriello, "Extracting Places from Traces of Locations," WMASH, October 1, 2004, pp 110-118.

[18] R. Hariharan, K. Toyama, "Project Lachesis: Parsing and Modeling Location Histories", $3^{\text {rd }}$ International Conference on Geographic Information Science, October 2004.

[19] J. Reades, et al, C. Ratti, "Cellular Census: Explorations in Urban Data Collection", IEEE Pervasive Computing, JulySeptember 2007, pp.30-38.

[20] J.J. Astrain, J. Villandangos, J.R. Garitagoitia, J.R. Gonzalez de Mendivil, "Fuzzy Location and Tracking on Wireless Networks", MobiWAC, October 2006, pp.84-91

[21] K. Derr, M. Manic, "Wireless Indoor Location Estimation Based on Neural Network RSS Signature Recognition (LENSR)", IEEE ICIEA08, The 3rd IEEE Conference on Industrial Electronics and Applications, June 3-5, 2008.

[22] K. Derr, M. Manic, "Intelligent Control in Automation Based on Wireless Traffic Analysis", 12th IEEE International Conference on Emerging Technologies and Factory Automation, Patras, Greece, September 2007.

[23] E. Cox, Fuzzy Modeling and Genetic Algorithms for Data Mining and Exploration, Morgan Kaufmann Publishers, 2005, pp. 95-147, 265-338.

[24] L. Tsoukalas, R. Uhrig, "Fuzzy and Neural Approaches in Engineering", Wile Interscience, 1997, pp. 145-188. 